Lim, L. H. I., Ye, Z., Ye, J., Yang, D., and Du, H. (2014) A linear method to extract diode model parameters of solar panels from a single I-V curve. Renewable Energy, 76. pp. 135-142. ISSN 0960-1481

Copyright (C) 2014 Elsevier Ltd.

A copy can be downloaded for personal non-commercial research or study, without prior permission or charge

Content must not be changed in any way or reproduced in any format or medium without the formal permission of the copyright holder(s)

When referring to this work, full bibliographic details must be given

http://eprints.gla.ac.uk/100845

Deposited on: 15 January 2015

Enlighten - Research publications by members of the University of Glasgow http://eprints.gla.ac.uk 


\title{
A linear method to extract diode model parameters of solar panels from a single I-V curve
}

\author{
Li Hong Idris Lima,*, Zhen $\mathrm{Ye}^{\mathrm{b}}$, Jiaying $\mathrm{Ye}^{\mathrm{c}}$, Dazhi Yang ${ }^{\mathrm{c}}$, Hui Du ${ }^{\mathrm{c}}$ \\ ${ }^{a}$ University of Glasgow, University of Glasgow (Singapore), 535 Clementi Road, Blk 47 \\ \#05-01, Singapore 599489 \\ ${ }^{b}$ REC Cells Pte Ltd., 20 Tuas South Avenue 14, Singapore 637312 \\ ${ }^{c}$ Solar Energy Research Institute of Singapore (SERIS), National University of Singapore \\ (NUS), Blk E3A \#06-01, 7 Engineering Drive 1, Singapore 117574
}

\begin{abstract}
The I-V characteristic curve is very important for solar cells/modules being a direct indicator of performance. But the reverse derivation of the diode model parameters from the $\mathrm{I}-\mathrm{V}$ curve is a big challenge due to the strong nonlinear relationship between the model parameters. It seems impossible to solve such a nonlinear problem accurately using linear identification methods, which is proved wrong in this paper. By changing the viewpoint from conventional static curve fitting to dynamic system identification, the integral-based linear least square identification method is proposed to extract all diode model parameters simultaneously from a single I-V curve. No iterative searching or approximation is required in the proposed method. Examples illustrating the accuracy and effectiveness of the proposed method, as compared to the existing approaches, are presented in this paper. The possibility of real-time monitoring of model parameters versus environmental factors (irradiance and/or temperatures) is also discussed.
\end{abstract}

Keywords: diode model, I-V curve, linear least square, binary search algorithm

\footnotetext{
${ }^{*}$ Corresponding author. Tel: +65 64690962

Email address: LiHonIdris.Lim@glasgow.ac.uk (Li Hong Idris Lim)
} 


\section{Introduction}

The current-voltage $(I-V)$ characteristics of solar cells/modules has the most importance in the photovoltaic industry because it exactly reflects the cell/module performance. However, it is not straightforward to get the model parameters from the $I-V$ curve for photovoltaic (PV) cells/modules [1]. For example, the $I-V$ relationship of the one-diode model as shown in Figure 1 is represented by

$$
I=I_{L}-I_{o}\left(\mathrm{e}^{\frac{V+R s I}{a}}-1\right)-\frac{V+R_{s} I}{R_{s h}},
$$

where $I_{L}$ is the photocurrent proportional to the irradiance, $I_{o}$ is the reverse saturation current of the diode, $a$ is the modified ideality factor [2], $R_{s}$ and $R_{s h}$ are resistance in series and parallel, respectively. Only $I$ and $V$ are known from the real measurement or datasheet.

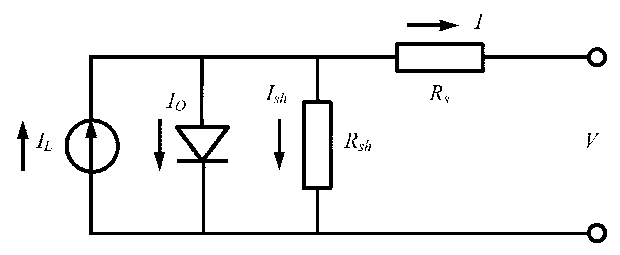

Figure 1: Equivalent circuit of one-diode model

In order to extract all the five unknown model parameters $\left(I_{L}, I_{o}, a, R_{s}\right.$ and $R_{s h}$ ) from the $I-V$ curve simultaneously, at least five independent pieces of information are required. To simplify the equation for easily solving, the following extreme conditions are utilised:

- Short circuit $(V=0)$ :

$$
\begin{gathered}
I_{s c}=I_{L}-I_{o}\left(\mathrm{e}^{\frac{R s I s c}{a}}-1\right)-\frac{R_{s} I_{s c}}{R_{s h}} . \\
\left.\frac{\mathrm{d} I}{\mathrm{~d} V}\right|_{s c} \approx-\frac{1}{R_{s h}} .
\end{gathered}
$$

- Open circuit $(I=0)$ :

$$
I_{L}-I_{o}\left(\mathrm{e}^{\frac{V o c}{a}}-1\right)-\frac{V_{o c}}{R_{s h}}=0,
$$


- Maximum power point (MPP):

$$
\begin{gathered}
I_{m p p}=I_{L}-I_{o}\left(\mathrm{e}^{\frac{V m p p+R s I m p p}{a}}-1\right)-\frac{V_{m p p}+R_{s} I_{m p p}}{R_{s h}}, \\
\left.\frac{\mathrm{d} I V}{\mathrm{~d} V}\right|_{m p p}=-\frac{V_{m p p}}{R_{s}+\frac{1}{\frac{I o}{a} \mathrm{e} \frac{V m p p+I m p p R s}{a}+\frac{1}{R_{s h}}}}+I_{m p p}=0 .
\end{gathered}
$$

10 Nevertheless, there are still no analytical solutions to (2)-(6) due to the highly nonlinearity inertially existed. Numerical solutions based on iterative searching algorithms with nonlinear least square have to be found, but it heavily depends on the initial values and is easy to be trapped by the local optimums [3, 4]. Moreover, for different initial value guess, such an approach can result in widely different parameter solutions, all leading to satisfactory curve fitting [5, 6 .

An alternative way to solving such a problem is to estimate the unknown parameters separately, which have been presented in several publications. Pelanchon et al. studies the influence of the structure parameters of a silicon solar cell on photocurrent, where $I_{L}$ can be estimated [7. Ravindra and Prasad reveals that $I_{o}$ is material independent and can be explicitly related to a solid state parameter, the $0 K$ Debye temperature of the semiconductor 8 . Singh et al. presents the use of properties of special trans function theory (STFT) for determining the ideality factor of real solar cell [9. Priyanka and Singh proposed a new method to determine $R_{s h}$ based on the single exponential model 25 and utilizing the steady state illuminated $I-V$ characteristics in third and fourth quadrants and the $V_{o c}-I_{s c}$ characteristics of the cell [10. Last but not the least, estimation methods for $R_{s}$ are well summarised in [11]. More recent methods in extracting $R_{s}$ are presented in [1, 6. All these methods have to assume some simplification, in order to achieve a balance between accuracy and calculation

so burden. Usually, more than one $I$ - $V$ curves are required because the biased $I-V$ characteristics are utilised in the estimation. They may work well for their respective single parameter estimation, but if the estimated parameters are put together into (1), the error becomes unacceptable $[5]$.

Nowadays, the prevailing modelling methods for PV cells/modules are more 35 or less based on(2)-(6), where only the three remarkable points (SC, OC, MPP) 
are utilized. This is insufficient to get accurate, unique and reliable model parameters. It is well known that the more information we know, the more accurate model parameters we can get. However, the PV manufacturers/institutes who have the facilities to obtain the full information of the entire $I$ - $V$ curves

40 often lack of an approach to utilize it, which is the method proposed in this paper.

This paper presents an all-new viewpoint of dynamic systems to solve the static equation (2)-(6). Note that for a first-order linear system described by the differential equation

$$
T \frac{\mathrm{d} y(t)}{\mathrm{d} t}+y(t)=u(t),
$$

where $T$ is the time constant, its unit ramp response is governed by

$$
y(t)=t+T\left(\mathrm{e}^{-\frac{t}{T}}-1\right),
$$

which has the same format (summation of exponentials and polynomials) as (1). This motivates us that the $I$ - $V$ curve represented by (1) can be viewed as the output of some linear system, and the parameters of one-diode model can 45 be linked to the parameters of a linear differential equation, which can then be well obtained by linear system identification methods [12, 13.

This whole paper is organised as follows. Section 2 describes the way to transform the static $I$ - $V$ curve to the dynamic linear system output. Integralbased system identification methods and linear least square algorithm are then so applied in Section 3 to get all the parameters of one-diode model simultaneously. Examples are given in Section 4 to illustrate the accuracy and effectiveness of the proposed method. A comparison with the nonlinear least square methods is demonstrated in Section 5. Outdoor module testing results are shown in Section 6 to demonstrate the reliability of the proposed method. Conclusions are given 55 in Section 7. 


\section{Dynamic system formulation}

Recall the $I-V$ curve represented by (1), let $y=I$ and $x=V+R_{s} I$, (1) then becomes,

$$
y=\left(I_{L}+I_{o}\right)-I_{o} \mathrm{e}^{\frac{x}{a}}-\frac{x}{R_{s h}} .
$$

Taking differential once on both sides of (7) results in

$$
\frac{\mathrm{d} y}{\mathrm{~d} x}=-\frac{I_{0}}{a} \mathrm{e}^{\frac{x}{a}}-\frac{1}{R_{s h}} .
$$

Taking a second differential for (8) then results in

$$
\frac{\mathrm{d}^{2} y}{\mathrm{~d} x^{2}}=-\frac{I_{o}}{a^{2}} \mathrm{e}^{\frac{x}{a}}
$$

By deleting $\mathrm{e}^{x / a}$ from $(\underline{8})$ and $(\underline{9})$,

$$
a \frac{\mathrm{d}^{2} y}{\mathrm{~d} x^{2}}-\frac{\mathrm{d} y}{\mathrm{~d} x}=\frac{1}{R_{s h}} .
$$

Let $t=x$ and $u(t) \equiv 1,(10)$ is equivalent to

$$
a \frac{\mathrm{d}^{2} y(t)}{\mathrm{d} t^{2}}-\frac{\mathrm{d} y(t)}{\mathrm{d} t}=\frac{u(t)}{R_{s h}}
$$

which is a standard differential equation representation of a second order linear system [14. $t$ denotes the "time", $u(t)$ and $y(t)$ are the system "input" and "output", respectively. Since $u(t) \equiv 1, y(t)$ is the unit step response of the system in "time" domain. Taking Laplace transform, $F(s)=\mathcal{L}[f(t)]=\int_{0}^{\infty} \mathrm{e}^{-s t} f(t) \mathrm{d} t$, on both sides of (11),

$$
a\left[s^{2} Y(s)-s y(0)-y^{\prime}(0)\right]-[s Y(s)-y(0)]=\frac{1}{R_{s h}} U(s) .
$$

Note from (7) and (8) that $y(0)=I_{L}, y^{\prime}(0)=-I_{o} / a-1 / R_{s h}$ and $s U(s)=1$, (12) is equivalent to

$$
a\left[s^{2} Y(s)-s^{2} U(s) y(0)-s U(s) y^{\prime}(0)\right]-[s Y(s)-s U(s) y(0)]=\frac{1}{R_{s h}} U(s) .
$$

Then the transfer function from $Y(s)$ to $U(s)$ is

$$
\begin{aligned}
G(s)=\frac{Y(s)}{U(s)} & =\frac{a y(0) s^{2}+\left[a y^{\prime}(0)-y(0)\right] s+\frac{1}{R_{s h}}}{a s^{2}-s} \\
& =\frac{a I_{L} s^{2}-\left(I_{o}+\frac{a}{R_{s h}}+I_{L}\right) s+\frac{1}{R_{s h}}}{a s^{2}-s} .
\end{aligned}
$$


The corresponding time domain differential equation is

$$
a \frac{\mathrm{d}^{2} y(t)}{\mathrm{d} t^{2}}-\frac{\mathrm{d} y(t)}{\mathrm{d} t}=a I_{L} \frac{\mathrm{d}^{2} u(t)}{\mathrm{d} t^{2}}-\left(I_{L}+I_{o}+\frac{a}{R_{s h}}\right) \frac{\mathrm{d} u(t)}{\mathrm{d} t}+\frac{u(t)}{R_{s h}} .
$$

It should be noted that (13) is different from (11) because all the initial conditions have been taken into account. So $(13)$ is the description of the same system as described by (11) but with zero initial conditions. This aids in the

60 proposed integral-based identification method, which will be shown as follows.

\section{Integral-based linear identification}

For an integer $m \geq 1$, define the multiple integral $[12$ as

$$
\int_{\left[t_{1}, t_{2}\right]}^{(m)} y(t)=\underbrace{\int_{t_{1}}^{t_{2}} \int_{t_{1}}^{\tau_{m}} \cdots \int_{t_{1}}^{\tau_{2}}}_{m} y\left(\tau_{1}\right) \mathrm{d} \tau_{1} \mathrm{~d} \tau_{2} \cdots \mathrm{d} \tau_{m-1} \mathrm{~d} \tau_{m} .
$$

Apply it to (13) with $t_{1}=0, t_{2}=t$ and $m=2$,

$$
a y(t)-a I_{L} u(t)+\left(I_{L}+I_{o}+\frac{a}{R_{s h}}\right) \int_{[0, t]}^{(1)} u(t)-\frac{1}{R_{s h}} \int_{[0, t]}^{(2)} u(t)=\int_{[0, t]}^{(1)} y(t) .
$$

Let $\gamma(t)=\int_{[0, t]}^{(1)} y(t)$ and

$$
\theta=\left[\begin{array}{c}
a \\
a I_{L} \\
\left(I_{L}+I_{o}+\frac{a}{R_{s h}}\right) \\
\frac{1}{R_{s h}}
\end{array}\right], \phi(t)=\left[\begin{array}{c}
y(t) \\
-u(t) \\
\int_{[0, t]}^{(1)} u(t) \\
-\int_{[0, t]}^{(2)} u(t)
\end{array}\right],
$$

then (14) can be rewritten as

$$
\phi^{T}(t) \theta=\gamma(t)
$$

Note that (15) holds for $t=t_{i}, i=1,2, \cdots, N$, where $N$ is the the number of data samples on the $I-V$ curve, it yields from (15) that

$$
\Phi \theta=\Gamma
$$


where $\Phi=\left[\phi\left(t_{1}\right), \phi\left(t_{2}\right), \cdots, \phi\left(t_{N}\right)\right]^{T}$ and $\Gamma=\left[\gamma\left(t_{1}\right), \gamma\left(t_{2}\right), \cdots, \gamma\left(t_{N}\right)\right]^{T}$. Thus, if $\Phi^{T} \Phi$ is nonsingular, the least square solution for $\theta$ by

$$
\theta=\left(\Phi^{T} \Phi\right)^{-1} \Phi^{T} \Gamma
$$

will minimise the following error

$$
\min _{\theta}(\Gamma-\Phi \theta)^{T}(\Gamma-\Phi \theta)
$$

The proof of the non singularity of $\Phi^{T} \Phi$ can be found in [12. Once $\theta$ is determined from (16), the parameters of one-diode model can be obtained by

$$
\begin{aligned}
a & =\theta_{1}, \\
I_{L} & =\frac{\theta_{2}}{\theta_{1}}, \\
I_{o} & =\theta_{3}-\frac{\theta_{2}}{\theta_{1}}-\theta_{1} \theta_{4}, \\
R_{s h} & =\frac{1}{\theta_{4}} .
\end{aligned}
$$

\subsection{Determination of $R_{s}$}

In order to calculate $\theta$ from (16), $\Phi$ and $\Gamma$ must be known. As both of them are integrals to $t, t$ must be prior known. However, $t=V+R_{s} I$, if $R_{s}$ cannot

${ }_{65}$ be determined, $\Phi$ and $\Gamma$ become unknown so that $\theta$ cannot be calculated from (16).

There are many ways to determine $R_{s}$ beforehand [1]. Recent developments are: (1) partial shading method [1]; and (2) local ideality method [6. All of them either need multiple $I-V$ curves or assume dominant exponential, which 70 is not suitable for our purpose here.

It is well known that $R_{s}$ impacts the shape of current and voltage curve near the maximum power point [2]. If $R_{s}$ increases, the MPP point will move to the right and the error between the real and estimated $I-V$ curves will be positive; If $R_{s}$ decreases, the MPP point will move to the left and the error between the real and estimated $I-V$ curves will be negative, as shown in Figure 2, Thus, 


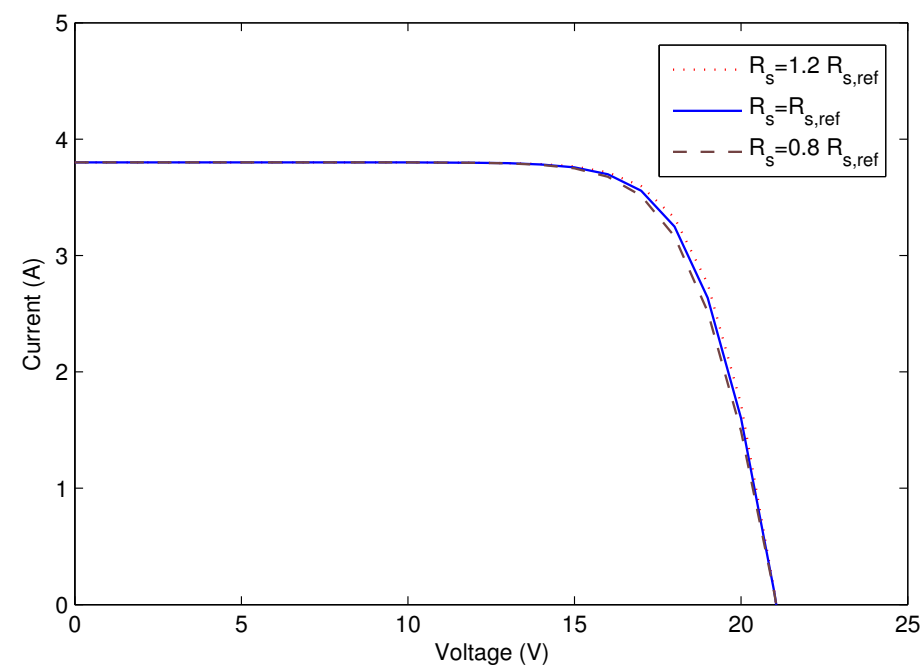

Figure 2: Effect of series resistance for a single crystalline cell at standard operating conditions.

$R_{s}$ can be used as a tuning parameter such that the root mean square error between the real and estimated $I-V$ curves is minimised, i.e.,

$$
\min _{R_{s}} \sqrt{\frac{1}{N} \sum_{i=1}^{N}\left[\hat{y}\left(t_{i}\right)-y\left(t_{i}\right)\right]^{2} .}
$$

It derives from (1) that

$$
\left.\frac{\mathrm{d} I}{\mathrm{~d} V}\right|_{o c}=-\frac{1}{R_{s}+\frac{1}{\frac{I o}{a} \mathrm{e}^{\frac{V o c}{a}}+\frac{1}{R_{s h}}}},
$$

so

$$
R_{s}+\frac{1}{\frac{I_{o}}{a} \mathrm{e}^{\frac{V o c}{a}}+\frac{1}{R_{s h}}}=-\frac{1}{\left.\frac{\mathrm{d} I}{\mathrm{~d} V}\right|_{o c}}>R_{s}
$$

which implies that $-1 /\left.\frac{\mathrm{d} I}{\mathrm{~d} V}\right|_{o c}$ is the upper bound of $R_{s}$, i.e., $R_{s}^{u p p}=-1 /\left.\frac{\mathrm{d} I}{\mathrm{~d} V}\right|_{o c}$. The lower bound of $R_{s}$ can be zero, i.e., $R_{s}^{\text {low }}=0$. With such a band of $R_{s}$, binary search algorithm is applied to determine $R_{s}$ in the following way:

Step 1. Arbitrarily choose $R_{s}$ from $\left[R_{s}^{l o w}, R_{s}^{u p p}\right]$ and calculate $\hat{a}, \hat{I}_{L}, \hat{I}_{o}$ and $\hat{R}_{s h}$ 75 from the proposed linear least square (16); 
Step 2. Calculate from (1) that

$$
\hat{y}(t)=\hat{I}_{L}-\hat{I}_{o}\left(\mathrm{e}^{\frac{V+R+R I}{\hat{a}}}-1\right)-\frac{V+R_{s} I}{\hat{R}_{s h}},
$$

and the root mean square error (RMSE)

$$
R M S E=\sqrt{\frac{1}{N} \sum_{i=1}^{N}\left[\hat{y}\left(t_{i}\right)-y\left(t_{i}\right)\right]^{2}} .
$$

Step 3. Calculate

$$
E R R=\sum_{i=1}^{N}\left[\hat{y}\left(t_{i}\right)-y\left(t_{i}\right)\right] .
$$

If $E R R>0$, it implies a bigger estimation of $R_{s}$. Otherwise, it implies a smaller estimation of $R_{s}$. Adjust $R_{s}$ as follows:

$$
R_{s}= \begin{cases}\frac{R_{s}+R_{s}^{\text {low }}}{2}, & \text { if } E R R>0 \\ \frac{R_{s}+R_{s}^{u p p}}{2}, & \text { otherwise. }\end{cases}
$$

Step 4. Update $R_{s}^{u p p}$ and $R_{s}^{\text {low }}$ according to the sign of $E R R$. If $E R R>0$, $R_{s}^{u p p}=R_{s}$, otherwise, $R_{s}^{\text {low }}=R_{s}$.

Step 5. If RMSE is less than some tolerance (Tol) or iterative cycle reaches some preset number (n), stop the searching. Otherwise, go back to Step 2.

The flowchart of the binary searching algorithm is shown in Figure 3 .

\section{Examples}

The $I-V$ characteristics of full-sized commercial modules were measured in the PV module testing lab of Solar Energy Research Institute of Singapore (SERIS). A pulsed solar simulator (PASAN IIIB) with a constant illumination intensity plateau of about $12 \mathrm{~ms}$ is used. The data acquisition, which requires about $10 \mathrm{~ms}$, occurs during the plateau period, whereby the light intensity varies by less than $\pm 1 \%$. The intensity of the solar simulator is calibrated with a c-Si reference cell certified by Fraunhofer ISE. If thin-film modules are measured, spectral mismatch correction is applied. The overall uncertainty of module 90 power measurement is within $\pm 2 \%$. 


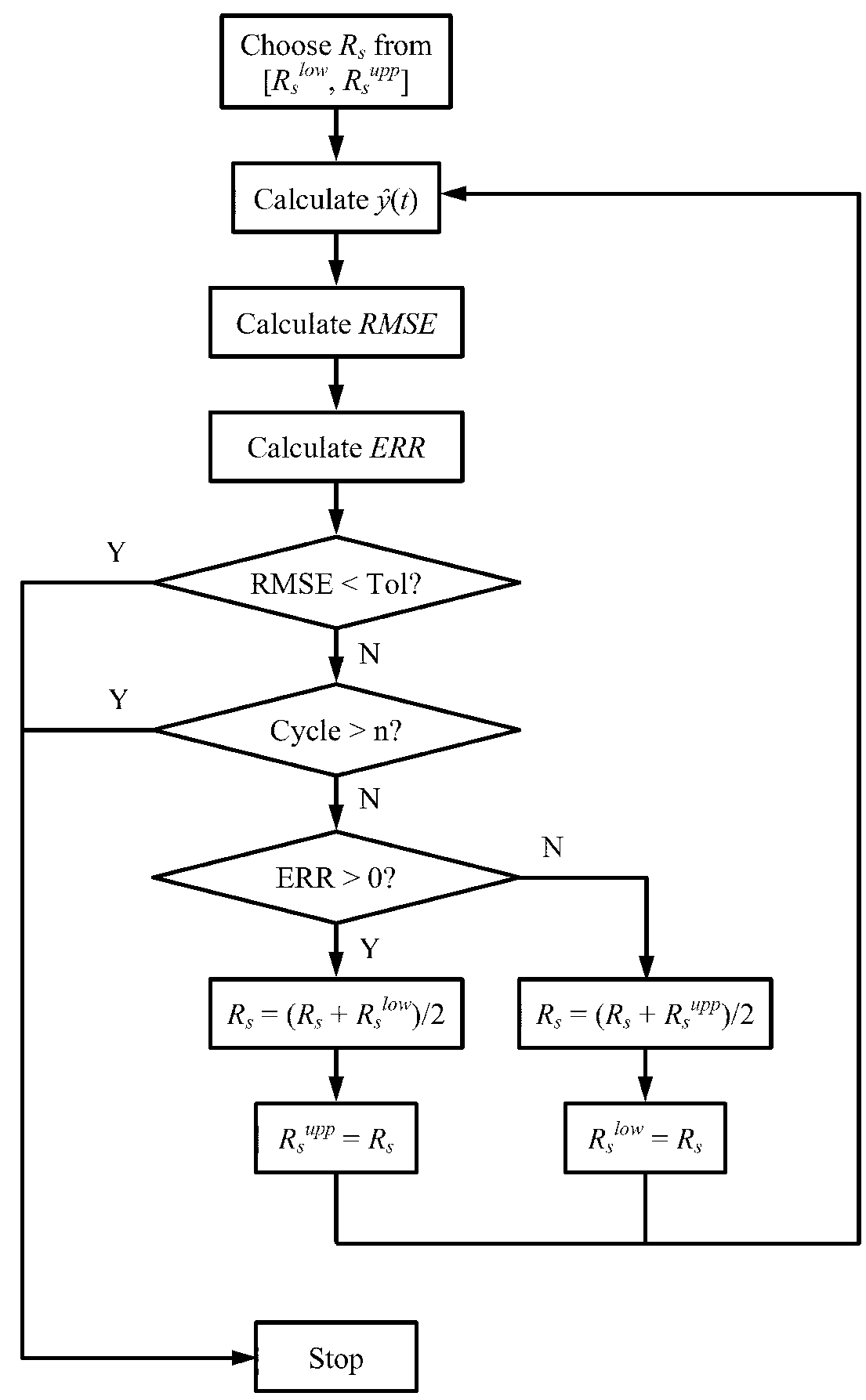

Figure 3: Flowchart of the binary searching algorithm 


\subsection{Example 1 (STC I-V characteristics of c-Si modules)}

The $I-V$ characteristic of a crystalline PV module under STC from the PV module testing lab of SERIS is shown in Figure 4 .

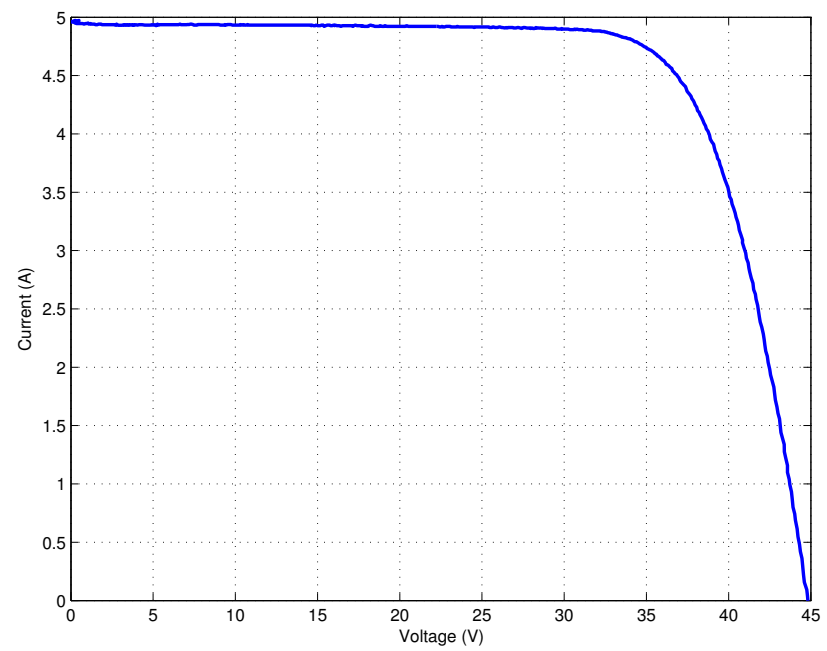

Figure 4: The $I-V$ characteristic of a crystalline PV module calibrated by SERIS

Firstly, the upper bound of $R_{s}(\Omega)$ is calculated by

$$
R_{s}^{u p p}=-\frac{1}{\left.\frac{\mathrm{d} I}{\mathrm{~d} V}\right|_{o c}}=1.0952 .
$$

Secondly, choose $R_{s}=1.0952$ and apply the proposed binary searching with Tol $=2 \%$. After the searching stops, $R_{s}=0.655$. It follows from (16) that

$$
\begin{aligned}
& \theta_{1}=1.9891, \\
& a=1.9891(\mathrm{~V}), \\
& \theta_{2}=9.8295 \text {, } \\
& I_{L}=4.9416(\mathrm{~A}) \text {, } \\
& \theta_{3}=4.9434 \text {, } \\
& I_{o}=4.1785 \times 10^{-9}(\mathrm{~A}) \text {, } \\
& \theta_{4}=8.9631 \times 10^{-4}, \\
& R_{s h}=1.1157 \times 10^{3}(\Omega) \text {. }
\end{aligned}
$$

The comparison between the real measured I-V curve and the fitted one are shown in Figure 5 .

Figure 6 illustrates the convergence of $R_{s}$ and $R M S E$. It is easy to see that $R_{s}$ and $R M S E$ converge fast within 30 searching steps only. 

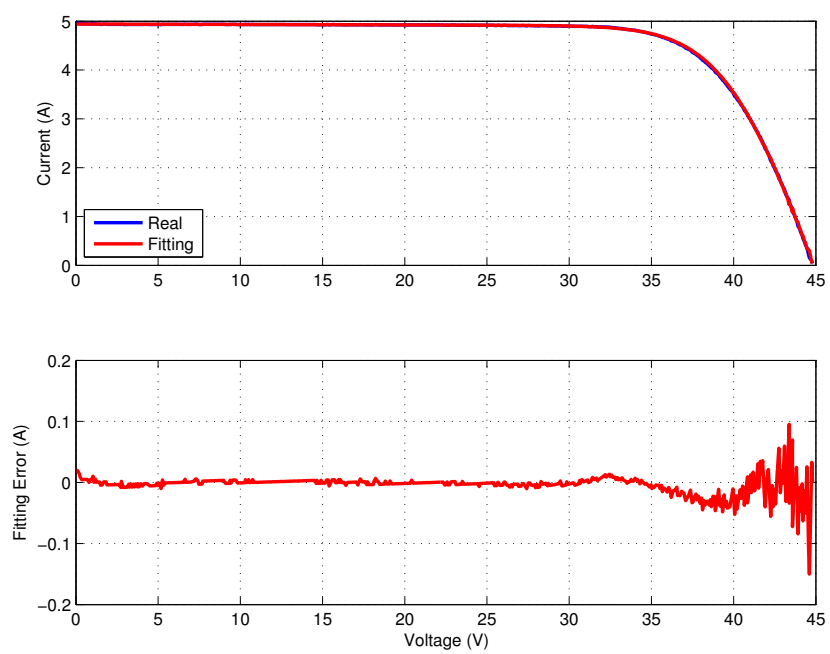

Figure 5: Comparison of the $I-V$ curve between measurement and estimation
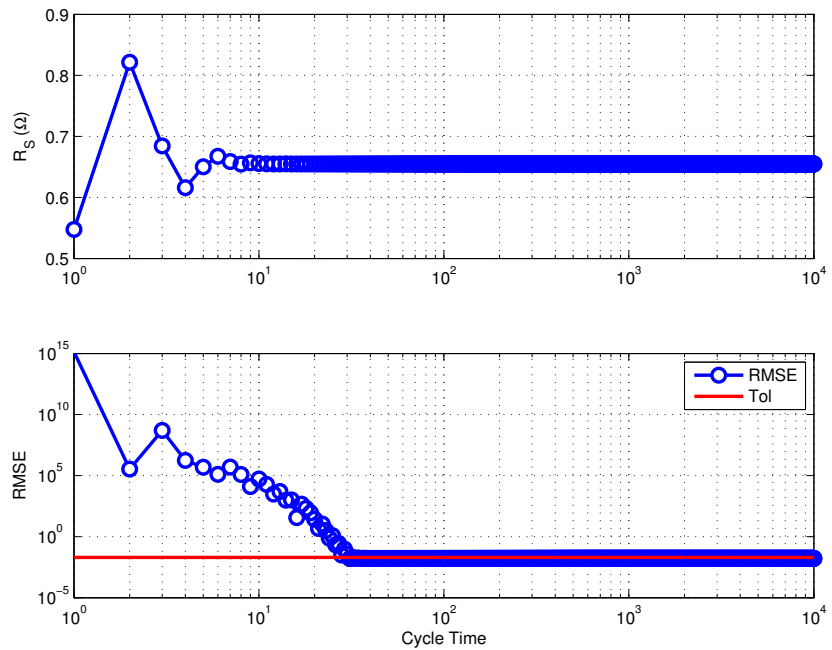

Figure 6: Convergence of $R_{s}$ and $R M S E$ in the binary searching 


\subsection{Example 2 (I-V characteristics family of c-Si modules)}

The $I-V$ characteristic family of a c-Si PV module under varying irradiance from $400 \mathrm{~W} / \mathrm{m}^{2}$ to $1200 \mathrm{~W} / \mathrm{m}^{2}$ is shown in Figure 7. Similar to Example 1, the flash test data is also obtained from the PV module testing lab of SERIS.

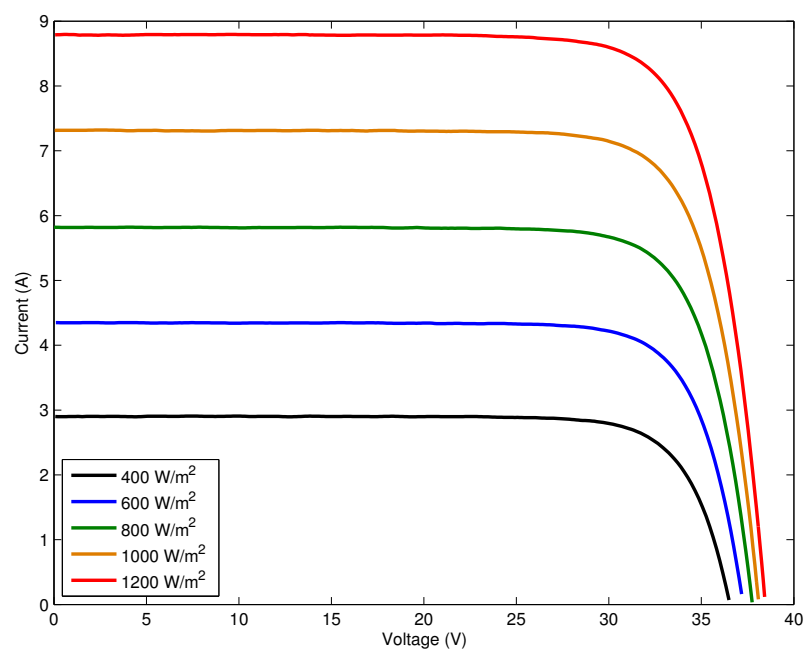

Figure 7: The $I-V$ characteristic of a thin-film PV module calibrated by SERIS

Following the same procedure as shown in Example 1, the model parameters are shown in Table 1. It is evident that $I_{L}$ increases with increasing irradiance. The other model parameters like $a, I_{o}, R_{s}$ and $R_{s h}$ generally agree with the trends from the outdoor monitoring tests in Section 6. However, $I_{o}$ is relatively constant as it is largely dependent on the module temperature, which is not controlled under normal lab indoor conditions. The comparison between the real measured I-V curve and the fitted one is shown in Figure 8 .

\section{Comparison with nonlinear least square}

For a comparison between the conventional method to the proposed linear approach, the nonlinear least square method is applied to solve $(2)-(6)$ for Example 1. 
Table 1: Results for I-V characteristics family $\left(25^{\circ} \mathrm{C}\right)$

\begin{tabular}{ccccccc}
\hline $\begin{array}{c}\text { Irradiance } \\
\left(\mathrm{W} / \mathrm{m}^{2}\right)\end{array}$ & $\begin{array}{c}R_{s h} \\
(\Omega)\end{array}$ & $\begin{array}{c}I_{L} \\
(\mathrm{~A})\end{array}$ & $\begin{array}{c}I_{o} \\
(\mathrm{~A})\end{array}$ & $\begin{array}{c}R_{s} \\
(\Omega)\end{array}$ & $\begin{array}{c}a \\
(\mathrm{~V})\end{array}$ & RMSE \\
\hline 400 & $2.6458 \times 10^{3}$ & 2.9042 & $6.085 \times 10^{-8}$ & 0.2052 & 1.8960 & 0.0174 \\
600 & $2.5658 \times 10^{3}$ & 4.3457 & $6.8896 \times 10^{-8}$ & 0.2376 & 1.8959 & 0.0253 \\
800 & $2.0222 \times 10^{3}$ & 5.8179 & $7.9233 \times 10^{-8}$ & 0.2514 & 1.9070 & 0.0313 \\
1000 & $1.1322 \times 10^{3}$ & 7.3118 & $1.2012 \times 10^{-7}$ & 0.2518 & 1.9457 & 0.0429 \\
1200 & $1.0141 \times 10^{3}$ & 8.7951 & $1.4433 \times 10^{-7}$ & 0.2634 & 1.9542 & 0.0441
\end{tabular}

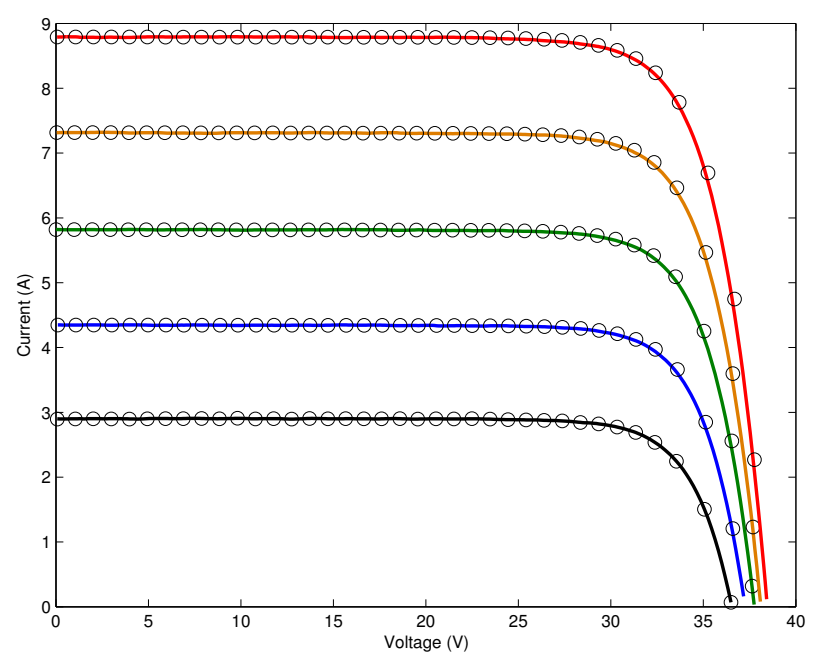

Figure 8: Comparison of the $I-V$ curve between measurement (straight line) and estimation (round markers) 
It follows from (3) that

$$
R_{s h} \approx-\frac{1}{\left.\frac{\mathrm{d} I}{\mathrm{~d} V}\right|_{s c}} .
$$

$\mathrm{d} I / \mathrm{d} V$ at short-circuit is estimation from the linear fit for: (i) $0<V<4$; (ii) $0<V<8$; (iii) $0<V<12$; (iv) $0<V<16$ (V). Initial values for the 115 other parameters are set to be: $I_{L}=0, I_{o}=0, R_{s}=0, a=1$. The results of nonlinear least square for the three different $R_{s h}$ are shown in Table 2 .

Table 2: Results from nonlinear least square

\begin{tabular}{cccccccc}
\hline Case & $R_{s h}(\Omega)$ & $I_{L}(\mathrm{~A})$ & $I_{o}(\mathrm{~A})$ & $R_{s}(\Omega)$ & $a(\mathrm{~V})$ & Cycles & $R M S E$ \\
\hline (i) & 178.2578 & 0.0257 & $-4.6053 \times 10^{-12}$ & -0.7825 & 1.8541 & 134 & 4.6771 \\
(ii) & 698.8465 & $7.6632 \times 10^{-4}$ & $-1.3278 \times 10^{-13}$ & -0.9394 & 1.7887 & 132 & 4.6306 \\
(iii) & $1.1802 \times 10^{3}$ & 4.9634 & $6.5754 \times 10^{-9}$ & 0.5653 & 2.1922 & 163 & 0.0265 \\
(iv) & $1.3184 \times 10^{3}$ & 4.9631 & $8.2778 \times 10^{-9}$ & 0.5550 & 2.2171 & 154 & 0.0288 \\
\hline
\end{tabular}

Iterations for Case (i) and (ii) have to be stopped because it is trapped by the local optimum (local singularity) and the step size is less than $10^{-12}$. Case (iii) and (iv) give the acceptable RMSE, but which one is the correct set of parameters for one-diode model?

This example shows the shortcomings of the conventional methods by solving $(2)-(\underline{6})$ with nonlinear least square:

- Only utilises the three points on the $I-V$ curves (open-circuit, short-circuit, MPP);

- Iteration convergence heavily depends on the initial conditions;

- Solution is not unique, i.e., different sets of parameters may results in the same acceptable fitting.

As compared to the nonlinear least square, the proposed method utilises the full points on the $I-V$ curve and provides the unique solution with a higher 130 accuracy. 


\section{Applications}

In Sections 4 and 5, the improved accuracy and solution uniqueness of the proposed method versus the conventional nonlinear least squares method, has been examined. In comparison to the lab measurement data in Sections 4 and

135 5, outdoor monitoring data exhibit dynamic behaviours under varying environmental conditions. In this section, the applicability of the proposed method for outdoor daily fluctuating conditions, will be illustrated.

\subsection{Outdoor module performance monitoring}

Outdoor module testing (OMT) is usually carried on by many PV panel manufacturers and research institutes for the module performance evaluation under the real environments. For example, a OMT facility has been setup by SERIS to measure the outdoor PV module performance in tropical Singapore, which is located in the campus of the National University of Singapore (NUS). The module under the OMT test was installed since August, 2010 with a fixed tilt angle of 10 facing south. DC parameters including full $I$ - $V$ curves, $V_{o c}, I_{s c}$, $V_{m p p}, I_{m p p}, P_{m p p}$ together with module temperature are measured and logged every minute. Environmental parameters including in-plane solar irradiance $G$, ambient temperature $T_{a m b}$, wind speed and wind direction are logged simultaneously with the DC parameters. Between $I-V$ measurements, electrical energy 150 is extracted from the module at its maximum power point (MPP). The uncertainty of all electrical measured parameters is within $\pm 0.1 \%$ for full scale. With these $I-V$ data in time series, the diode model parameters can be identified by the proposed method and correlated to the environmental factors like irradiance, temperature, etc. This helps improvements of module design by linking the environmental factors into diode model parameters directly.

Figure9 shows the time series of irradiance $\left(G_{s i}\right)$, ambient temperature $\left(T_{a m b}\right)$ and module temperature on the back sheet $\left(T_{m o d}\right)$ on a typical day from SERIS' OMT testbed. The plot is centered around the solar noon, which was at 13:10 on the 5th August 2010. 


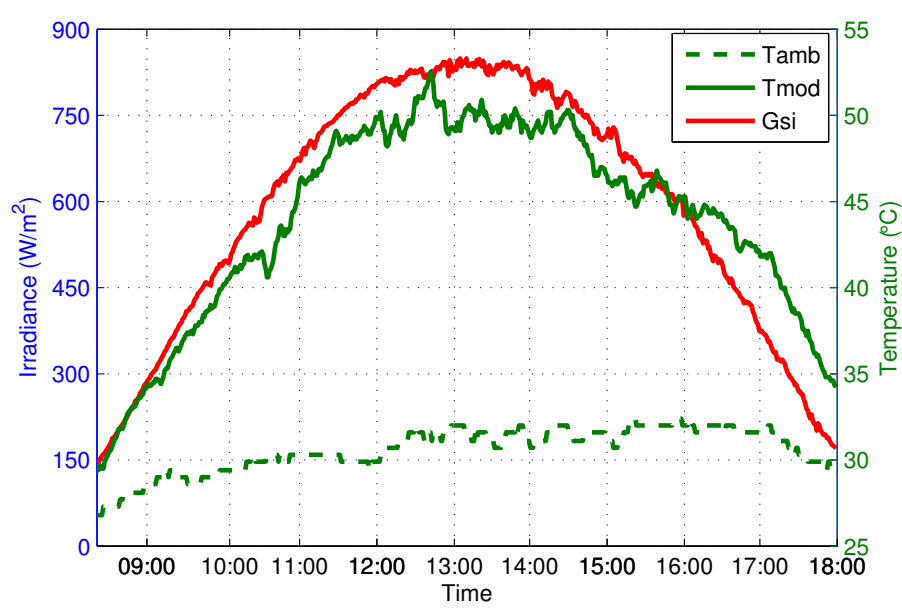

Figure 9: Environmental factors of a typical day in SERIS' OMT testbed

By applying the proposed method in Section 3 , the time-varying one-diode model parameters $I_{L}, I_{o}, a, R_{s}$ and $R_{s h}$ for the same day are identified, as shown in Figure10. The variation of the identified parameters in the one-diode model in Figure 10 reflects the dynamics of the PV module under different environmental operating conditions, which cannot be easily interpreted from the $I-V$ curve at each time instant.

The relationships between the identified parameters and the environmental operating conditions are further illustrated in Figures 11 to 14. A proportional relationship between $I_{L}$ and irradiance intensity is observed in Figure 11, It is also apparent that $I_{o}$ generally shows an increasing trend with rising module temperature [15, as shown in Figure 12. This also agrees with the theoretical temperature dependence of $I_{o}$, as given by

$$
I_{o}=B T^{3} \mathrm{e}^{-\frac{E g}{k T}}
$$

, where $E_{g}$ is the band gap of silicon and $B$ is a temperature independent constant [6].

Figure 13 illustrates that $a$ generally decreases with increasing irradiance for $G_{s i}<300 \mathrm{~W} / \mathrm{m}^{2}$ and increases beyond that, which is as reported in [16. 

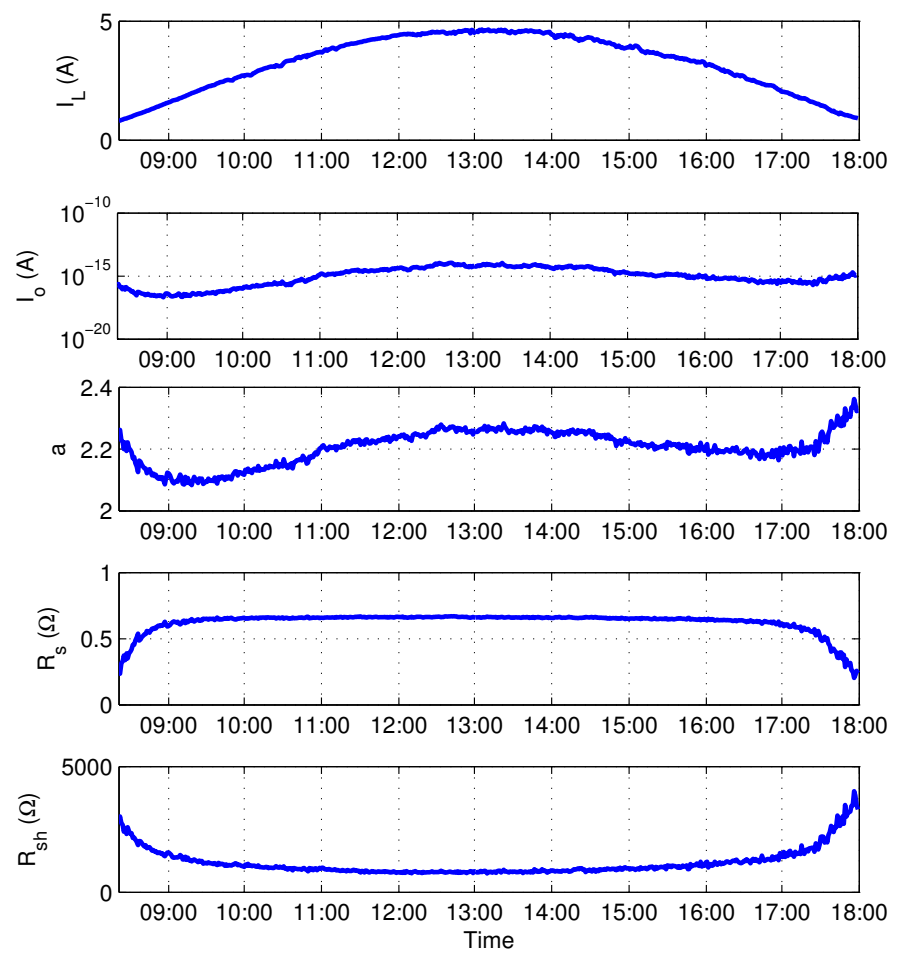

Figure 10: Identified one-diode model parameters

${ }_{170}$ When irradiance decreases in Figure 14, the series resistance $R_{s}$ decreases and the shunt resistance $R_{s h}$ increases, which is consistent with previous reported results [17. The decrease in $R_{s}$ is due to the decreased thermal loss $\left(I^{2} R_{s}\right)$ with decreasing irradiance.

\section{Conclusion}

A novel method is proposed in this paper to identify all the one-diode model parameters of PV panels from a single $I-V$ curve in one instance. As compared to the existing methods, the proposed approach is linear, simpler to be implemented and much more accurate without any approximation. It establishes the direct link between model parameters and $I-V$ curves, which provides engineers with a clear knowledge on how the PV cell/module is affected by the irradiance 


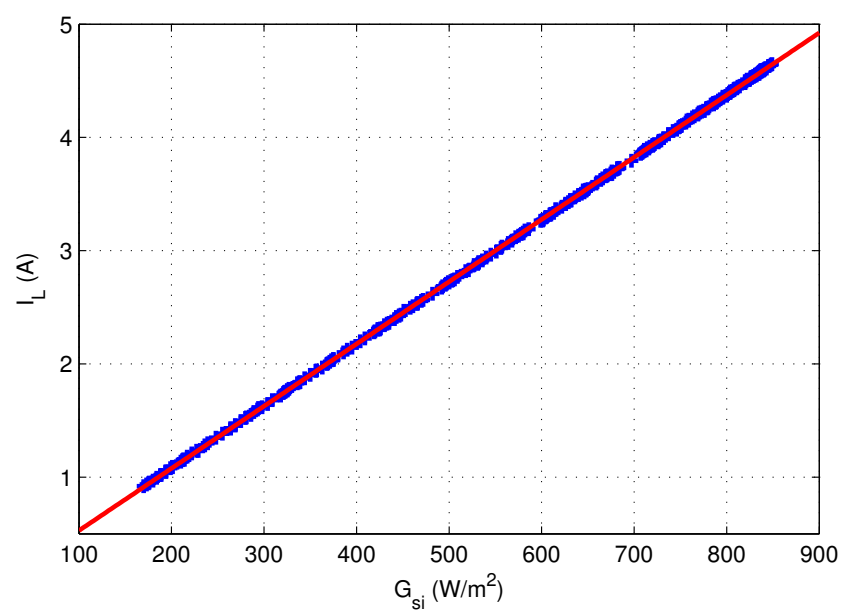

Figure 11: Proportional relationship between $I_{L}$ and $G_{s i}$

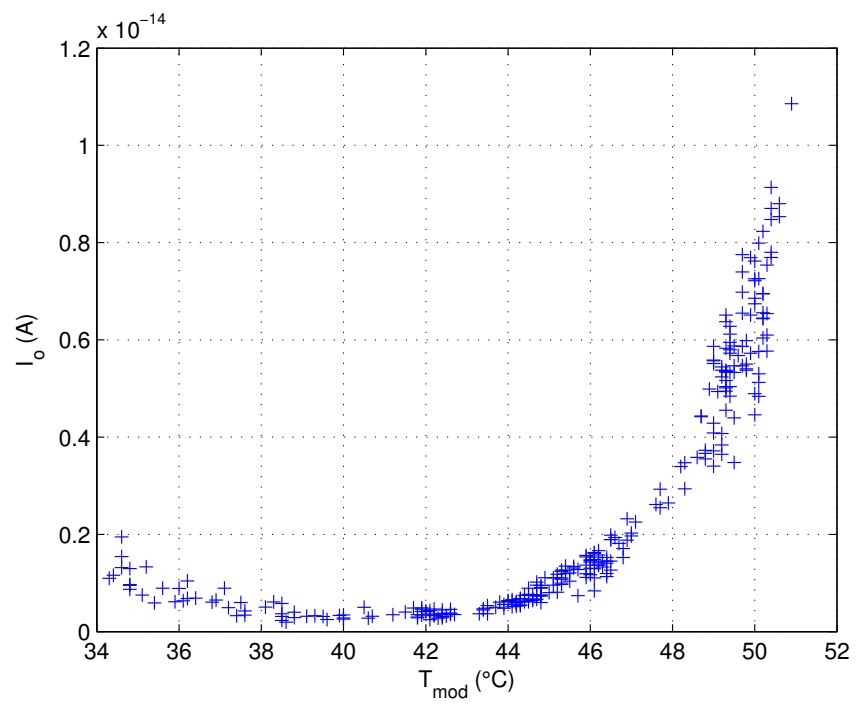

Figure 12: Relationship between $I_{o}$ and $T_{\text {mod }}$

and temperature. This is of importance in PV cell design and module assembly. 


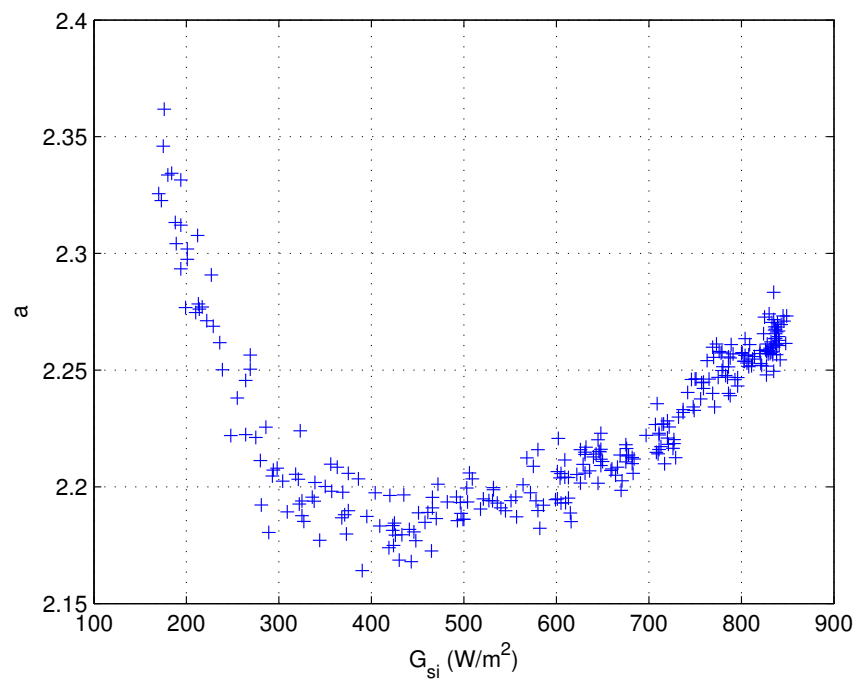

Figure 13: Relationship between $a$ to $G_{s i}$
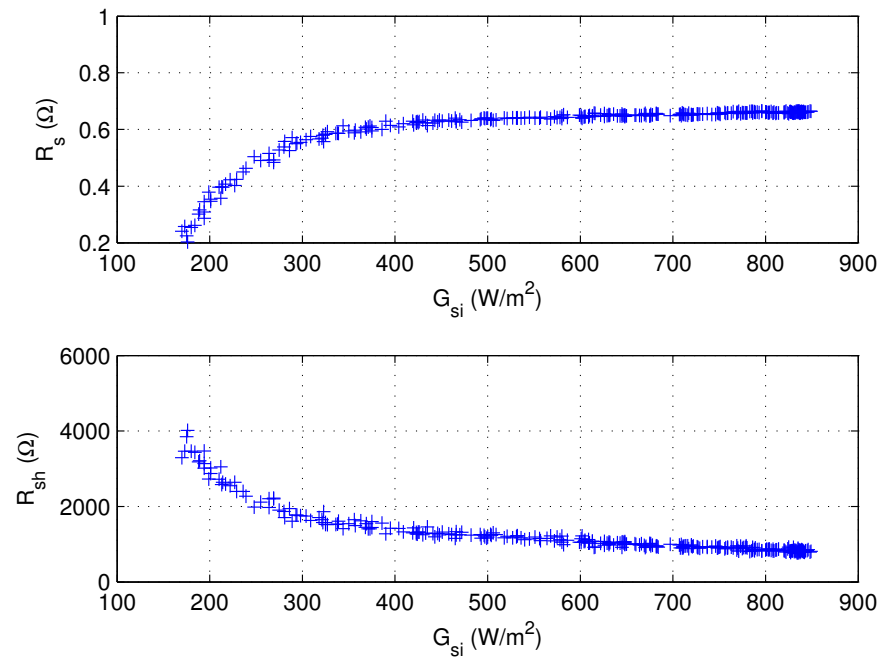

Figure 14: Relationship between $R_{s}, R_{s} h$ and $G_{s i}$

\section{References}

[1] Y. S. Kim, S.-M. Kang, B. Johnston, R. Winston, A novel method to extract the series resistances of individual cells in a photovoltaic module, 
[2] W. D. Soto, S. Klein, W. Beckman, Improvement and validation of a model for photovoltaic array performance, Solar Energy 80 (1) (2006) 78 - 88.

[3] J. R. Hauser, Numerical Methods for Nonlinear Engineering Models, Springer, 2009.

[4] C. T. Kelley, Iterative methods for optimization, SIAM, Philadelphia, 1999.

[5] J. Appelbaum, A. Chait, D. Thompson, Parameter estimation and screening of solar cells, Prog. Photovolt: Res. Appl. 1 (2) (1993) 93 - 106.

[6] G. H. Yordanov, O.-M. Midtgård, T. O. Saetre, Series resistance determination and further characterization of c-si PV modules, Renewable Energy 46 (10) (2012) $72-80$.

[7] F. Pelanchon, P. Mialhe, J. Charles, The photocurrent and the open-circuit voltage of a silicon solar cell, Solar Cells 28 (1) (1990) $41-55$.

[8] N. Ravindra, B. Prasad, Saturation current in solar cells: an analysis, Solar Cells 2 (2) (1980) $109-113$.

[9] N. S. Singh, A. Jain, A. Kapoor, Determination of the solar cell junction ideality factor using special trans function theory (STFT), Solar Energy Materials and Solar Cells 93 (8) (2009) 1423 - 1426.

[10] Priyanka, M. Lal, S. Singh, A new method of determination of series and shunt resistances of silicon solar cells, Solar Energy Materials and Solar Cells $91(2-3)(2007) 137-142$.

[11] D. Pysch, A. Mette, S. Glunz, A review and comparison of different methods to determine the series resistance of solar cells, Solar Energy Materials and Solar Cells 91 (18) (2007) 1698 - 1706.

[12] Q.-G. Wang, Y. Zhang, Robust identification of continuous systems with dead-time from step responses, Automatica 37 (3) (2001) 377 - 390. 
[13] M. Liu, Q.-G. Wang, B. Huang, C. C. Hang, Improved identification of continuous-time delay processes from piecewise step tests, Journal of Process Control 17 (1) (2007) $51-57$.

[14] R. B. R.C. Dorf, Modern Control Systems, Prentice Hall, 2008.

${ }_{215}$ [15] C. Hu, R. White, Solar cells: from basics to advanced systems, McGraw Hill Series in Electrical and Computer Engineering, McGraw-Hill, 1983.

[16] M. Hamdy, R. Call, The effect of the diode ideality factor on the experimental determination of series resistance of solar cells, Solar Cells 20 (2) (1987) $119-126$.

220 [17] M. C. Di Piazza, G. Vitale, Photovoltaic Sources: Modeling and Emulation, Springer, 2012. 